\title{
Hybrid Multicriteria Group Decision Making Method for Information System Project Selection Based on Intuitionistic Fuzzy Theory
}

\author{
Jian Guo \\ School of Management Science and Engineering, Central University of Finance and Economics, Beijing 100081, China \\ Correspondence should be addressed to Jian Guo; txmguojian2002@126.com
}

Received 10 August 2013; Revised 18 November 2013; Accepted 19 November 2013

Academic Editor: Hao-Chun Lu

Copyright (C) 2013 Jian Guo. This is an open access article distributed under the Creative Commons Attribution License, which permits unrestricted use, distribution, and reproduction in any medium, provided the original work is properly cited.

\begin{abstract}
Information system (IS) project selection is of critical importance to every organization in dynamic competing environment. The aim of this paper is to develop a hybrid multicriteria group decision making approach based on intuitionistic fuzzy theory for IS project selection. The decision makers' assessment information can be expressed in the form of real numbers, intervalvalued numbers, linguistic variables, and intuitionistic fuzzy numbers (IFNs). All these evaluation pieces of information can be transformed to the form of IFNs. Intuitionistic fuzzy weighted averaging (IFWA) operator is utilized to aggregate individual opinions of decision makers into a group opinion. Intuitionistic fuzzy entropy is used to obtain the entropy weights of the criteria. TOPSIS method combined with intuitionistic fuzzy set is proposed to select appropriate IS project in group decision making environment. Finally, a numerical example for information system projects selection is given to illustrate application of hybrid multi-criteria group decision making (MCGDM) method based on intuitionistic fuzzy theory and TOPSIS method.
\end{abstract}

\section{Introduction}

Information system (IS) project selection is of critical importance for every organization in rapid changing and dynamic technological environment [1]. In order to meet organization's strategic goals and operational objectives, IS project selection for business development need has become an important strategic and operational decision issue [2]. Selecting the most suitable from numerous competing proposals has an enormous impact on the productivity of the organization [3]. However, the process of evaluating and selecting IS projects is complex and challenging. It often involves multiple decision makers, multiple selection criteria, and subjective and imprecise assessments. A lot of quantitative and qualitative factors should be considered, such as outside environment conditions, business goals, initial investment, benefits, project risks, management ability of the organization and limited availability of IS resources. The assessment information given by the decision makers (or experts) over the IS project alternatives under each criterion may not be all described by exact numbers and sometimes they may take the following forms, such as exact numerical values, interval numbers, triangular fuzzy numbers, linguistic labels, or even intuitionistic fuzzy numbers (IFNs). The importance of the decision makers can all be expressed as linguistic variables such as very good, good, fair, poor, and very poor. So it is a hybrid multicriteria group decision making (MCGDM) problem which combines the multicriteria decision making (MCDM) and the group decision making (GDM) method for IS project selection.

MCGDM is one of the most common activities in modern society, which involves selecting the optimal one from a finite of alternatives with respect to a collection of the predefined criteria by a group of experts with a high collective knowledge level on these particular criteria. Various methodologies have been suggested by IS researchers to help organizations make good IS project selection decisions. The existing methods for IS project selection include single criterion cost-benefit analysis [4], multiple criteria scoring models [5] and ranking methods [6], subjective committee evaluation methods [7], or some other quantitative methods using Analytical Hierarchy Process (AHP) [8], goal programming [9], or 
DEA model [10]. Lee and Kim [11] suggested an integrated approach for interdependent IS project selection problems using Delphi, analytic network process concept and zero \pm one goal programming. Wu [12] proposed an effective IT project selecting method, which combined the decision making trial and evaluation laboratory (DEMATEL) with the analytic network process (ANP) and the zero-one goal programming (ZOGP) considering both financial and nonfinancial factors. Sometimes it is more convenient to use linguistic terms to evaluate the importance of decision makers and performance of alternatives. Fuzzy-based approach has been used to evaluate the information systems project due to its simplicity to deal with ambiguities and incomplete information [13]. The concept of a fuzzy set, introduced by Zadeh in 1965 [14], focuses on handling inexact and vague information that is intrinsic to natural language in terms of linguistic variables [15]. Chen and Cheng [3] presented a linguistic multicriteria decision making method (MCDM) for selecting an information, system project based on the fuzzy measure and the fuzzy integral. Yeh et al. [2] presented a fuzzy multicriteria group decision making approach for selecting information systems projects and proposed a multicriteria decision support system.

In the process of IS project decision making, a decision maker (DM) may not be able to accurately express his/her preferences for alternatives due to that DM may not possess a precise level of knowledge and the DM is unable to express the degree to which one alternative is better than others. In such cases, the DM may provide his/her preferences with a degree of doubt. The concept of intuitionistic fuzzy sets (IFSs) introduced by Atanassov is an extension of the classical fuzzy set theory [16], which are suitable way to deal with vagueness under uncertain environment. So far intuitionistic fuzzy sets (IFSs) have been used in many fields such as medical diagnosis [17], decision-making problems $[18,19]$, pattern recognition [20, 21], supplier selection [22], enterprise partners selection [23], and personnel selection [24]. Boran et al. [22] proposed a multicriteria intuitionistic fuzzy group decision making approach for supplier selection combined with TOPSIS method. Zhang and Liu [24] presented an intuitionistic fuzzy multicriteria group decision making method with grey relational analysis for personnel selection. Gerogiannis et al. [25] evaluated the project and portfolio management information systems using a combined intuitionistic fuzzy set-TOPSIS method, which involved both users and evaluators in the decision making process and tries to exploit the interest of each one in order to strengthen the final evaluation results. In the previously mentioned literature, the characteristics of the alternatives with respect to a set of criteria are represented by IFSs. However, requiring decision makers to express their opinions against alternatives as IFSs is not always appropriate due to the variety and complexity of decision-making problems. In most practical cases, it is more convenient and reasonable for a DM to express the criteria values of alternatives in different data types, such as exact values, intervals, intuitionistic fuzzy values (IFVs), and linguistic terms, naturally producing a hybrid MCGDM problem.
Several different transformation techniques have been developed for converting hybrid decision matrix into a unified form that needs to be further handled to rank alternatives. Liang et al. [26] studied the hybrid multiple attribute decision-making problem (MADM) in which the information about attribute weights was completely unknown. They turned a hybrid decision matrix with intervals and fuzzy values into a real decision matrix. Then a model was presented to seek objective weight based on entropy and subjective weight and objective weight were integrated into general weight. Wang and Cui [27] investigated a hybrid multiple attribute decision making problem with precision number, interval number, and fuzzy number. Herrera et al. [28] developed a fuzzy evaluation schema to deal with heterogeneous information and presented a transformation function which can unify linguistic, numerical, and interval valued information into the common format of 2-tuple fuzzy linguistic representation. Martínez et al. [29] developed an aggregation process for dealing with nonhomogeneous information composed of numerical, interval valued and linguistic information and took the 2-tuple fuzzy linguistic representation model as a base model. Si and Wei [30] introduced a hybrid multiattribute decision making method which transformed a hybrid decision matrix with exact values, intervals and linguistic terms into an intuitionistic fuzzy decision matrix and proposed an intuitionistic fuzzy optimization model to obtain the comprehensive evaluation value of each alternative, which is represented by intuitionistic fuzzy numbers. Liang et al. [31] proposed a new TOPSIS decision making approach for hybrid multiattribute group decision making problem with linguistic information and intuitionistic fuzzy values, they defined a new conversion function to transfer multigranularity linguistic information into IFNs. Guo and Li [32] developed an attitudinal-based method for constructing intuitionistic fuzzy information according to the attribute values expressed in different data types in hybrid MADM. Chen et al. [33] proposed an approach based on fuzzy preference relationship to solve the multiattribute decision making problem whose attribute weights were known and attribute values included real number, interval number, and fuzzy number.

In many practical situations, the decision makers for IS project selection may come from different departments and thus have different backgrounds and levels of knowledge, skills, experiences, and personalities. They may not have enough expertise or possess a sufficient level of knowledge to precisely express their preferences over the IS projects, and then they usually have some uncertainty in providing their preferences, which make the assessment information exhibits the characteristics of affirmation, negation, and hesitation. The evaluation information of the decision makers for IS project may be expressed in hybrid data forms such as real numbers, interval-valued numbers, linguistic variables, and IFNs. Converting hybrid data in MCGDM under uncertainty into IFVs is clearly significant because the flexibility in handling vagueness or uncertainty of the latter can avoid the loss and distortion of the original decision information and thus guarantee the mildness of fuzzy MCGDM and the reliability of the final decision results. The key to settle the 
question lies in an effective technique for fusing intuitionistic fuzzy information according to the evaluation information of hybrid decision matrix. In the process of aggregating the intuitionistic fuzzy information, both the weights of decision makers and the weights of criteria should be considered. However, the existing related research above does not focus on this aspect. Thus, these studies are inflexible and highly limited in practical applications.

In this paper we present an effective MCGDM approach to the IS project selection based on intuitionistic fuzzy theory. The decision makers' assessment information can be expressed in the form of real numbers, interval-valued numbers, linguistic variables, and IFNs. To model the subjectiveness and imprecision of the human decision making process, the importance of the decision makers is expressed in linguistic terms. All these evaluation information can be transformed to the form of intuitionistic fuzzy numbers. In Section 2, we give a brief review of basic concepts of intuitionistic fuzzy sets, then a conversion technique between real numbers, interval numbers, linguistic variables, and IFNs is introduced and a formula of intuitionistic fuzzy entropy is given to compute the importance of criteria. In Section 3, we outline a hybrid MCGDM model to select IS project based on the intuitionistic fuzzy theory and TOPSIS method. In Section 4, a numerical example is presented to illustrate the proposed approach and the comparisons have been made between the proposed method and other methods based on intuitionistic fuzzy theory to demonstrate the feasibility and practicality of the proposed method. Finally, some concluding remarks are pointed out.

\section{Intuitionistic Fuzzy Sets and Related Knowledge}

\subsection{Related Definitions of IFSs}

Definition 1 (see [16]). Let $X$ be a universe of discourse. An intuitionistic fuzzy set $A$ in $X$ is an object having the following form:

$$
A=\left\{\left\langle x, \mu_{A}(x), v_{A}(x)\right\rangle \mid x \in X\right\}
$$

where the functions $\mu_{A}(x)$ and $v_{A}(x)$ denote, respectively, the degree of membership and degree of nonmembership of the element $x \in X$ to the set $A \subseteq X$ with the condition that

$$
\begin{gathered}
0 \leq \mu_{A}(x) \leq 1, \quad 0 \leq v_{A}(x) \leq 1, \\
0 \leq \mu_{A}(x)+v_{A}(x) \leq 1 .
\end{gathered}
$$

One calls a third parameter $\pi_{A}(x)$ the intuitionistic index of the element $x$ in the set $A$. Consider the following:

$$
\pi_{A}(x)=1-\mu_{A}(x)-v_{A}(x) .
$$

It is the degree of indeterminacy membership of the element $x \in X$ to the set $A$. It is obvious that for every $x \in X, 0 \leq$ $\pi_{A}(x) \leq 1$.
Let $\alpha=\left(\mu_{\alpha}, v_{\alpha}\right), \beta=\left(\mu_{\beta}, v_{\beta}\right)$ be two intuitionistic fuzzy numbers, $\lambda>0$, then the operators can be defined as follows [34]:

$$
\begin{aligned}
& \text { (1) } \alpha \oplus \beta=\left(\mu_{\alpha}+\mu_{\beta}-\mu_{\alpha} \cdot \mu_{\beta}, v_{\alpha} v_{\beta}\right), \\
& \text { (2) } \alpha \otimes \beta=\left(\mu_{\alpha} \mu_{\beta}, v_{\alpha}+v_{\beta}-v_{\alpha} \cdot v_{\beta}\right), \\
& \text { (3) } \lambda \alpha=\left(1-\left(1-\mu_{\alpha}\right)^{\lambda}, v_{\alpha}^{\lambda}\right), \\
& \text { (4) } \alpha^{\lambda}=\left(\mu_{\alpha}^{\lambda}, 1-\left(1-v_{\alpha}\right)^{\lambda}\right) .
\end{aligned}
$$

Definition 2 (see [35]). Let $\alpha_{i}=\left(\mu_{i}, v_{i}\right)(i=1,2, \ldots, n)$ be a collection of intuitionistic fuzzy values, the intuitionistic fuzzy weighted averaging operator is defined as

$$
\begin{aligned}
& \operatorname{IFWA}_{\omega}\left(\alpha_{1}, \alpha_{2}, \ldots, \alpha_{n}\right) \\
& \quad=\omega_{1} \alpha_{1} \oplus \omega_{2} \alpha_{2} \oplus \cdots \oplus \omega_{n} \alpha_{n} \\
& =\left(1-\prod_{i=1}^{n}\left(1-\mu_{i}\right)^{\omega_{i}}, \prod_{i=1}^{n} v_{i}{ }^{\omega_{i}}\right),
\end{aligned}
$$

where $\omega_{i}$ is the weight of $\alpha_{i}, \omega_{i} \in[0,1]$ and $\sum_{i=1}^{n} \omega_{i}=1$.

Definition 3 (see [36]). A real function $d: \operatorname{IFS}_{S}(X) \rightarrow$ $[0,1]$ is called a distance for $\operatorname{IFS}_{S}$, if $d$ satisfies the following properties:

(1) $0 \leq d(A, B) \leq 1$

(2) $d(A, B)=0$ if and only if $A=B$, for all $A, B \in$ $\operatorname{IFS}_{S}(X)$

(3) $d(A, B)=d(B, A)$, for all $A, B \in \operatorname{IFS}_{S}(X)$;

(4) if $A \subseteq B \subseteq C$, then $d(A, C) \geq \max (d(A, B), d(B, C))$, for all $A, B, C \in \operatorname{IFS}_{S}(X)$.

Definition 4 (see [36]). Suppose that $A$ and $B$ are two intuitionistic fuzzy sets in $X, X=\left(x_{1}, x_{2}, \ldots, x_{n}\right)$. The Hamming distance between intuitionistic fuzzy sets $A$ and $B$ is $d_{\mathrm{IFS}}(A, B)$. Consider the following:

$$
\begin{aligned}
d_{\mathrm{IFS}}(A, B)=\frac{1}{2}( & \left|\mu_{A}\left(x_{i}\right)-\mu_{B}\left(x_{i}\right)\right|+\left|v_{A}\left(x_{i}\right)-v_{B}\left(x_{i}\right)\right| \\
& \left.+\left|\pi_{A}\left(x_{i}\right)-\pi_{B}\left(x_{i}\right)\right|\right) .
\end{aligned}
$$

2.2. Intuitionistic Fuzzy Transformation Techniques. Consider a hybrid multicriteria group decision making problem involving four different data types: exact values, intervals, IFVs, and linguistic terms. Let $A=\left\{A_{1}, A_{2}, \ldots, A_{n}\right\}$ be a finite set of alternatives and $C=\left\{c_{1}, c_{2}, \ldots, c_{m}\right\}$ be a set of criteria with a weight vector $w=\left(w_{1}, w_{2}, \ldots, w_{m}\right)^{T}$, where $w_{j} \geq 0(j=1,2, \ldots, m)$ and $\sum_{j=1}^{m} w_{j}=1$. Let $R^{(k)}=\left(a_{i j}^{(k)}\right)_{n \times m}$ be a hybrid decision matrix, where $a_{i j}^{(k)}$ can be exact values, intervals, IFVs, or linguistic terms. In terms of the ideas previously mentioned, we need to transform three other types of criteria values in $R^{(k)}$ into unified IFVs. In the following 
discussion, we will explore the transformation techniques for each of the aforementioned data types.

(1) Conversion between exact values and IFNs. The values of different criteria have different dimensions. Thus, the real numbers in the hybrid decision need to be standardized in order to eliminate interference in the final results.

Generally there are two kinds of criteria, the benefit type and the cost one. The higher the value of the benefit type is, the better it is. While in the cost type, it is the opposite.

For the benefit type, the standardizing formulae are listed as follows:

$$
b_{i j}^{(k)}=\frac{a_{i j}^{(k)}}{\sqrt{\sum_{i=1}^{m}\left(a_{i j}^{(k)}\right)^{2}}} .
$$

For the cost type, the standardizing formulae are listed as follows:

$$
b_{i j}^{(k)}=\frac{\left(1 / a_{i j}^{(k)}\right)}{\sqrt{\sum_{i=1}^{m}\left(1 / a_{i j}^{(k)}\right)^{2}}} .
$$

Standardized precise numbers can be transformed into intuitionistic fuzzy numbers $\alpha_{i j}^{(k)}=\left(\mu_{i j}^{(k)}, v_{i j}^{(k)}, \pi_{i j}^{(k)}\right)$. Consider the following:

$$
\mu_{i j}^{(k)}=b_{i j}^{(k)}, \quad v_{i j}^{(k)}=1-b_{i j}^{(k)}, \quad \pi_{i j}^{(k)}=0 .
$$

(2) Conversion between intervals and IFNs. We can use the formulae which are listed as follows to make conversion between intervals and IFNs.

For the benefit type, the standardizing formulae are listed as follows:

$$
\begin{aligned}
& b_{i j}^{L^{(k)}}=\frac{a_{i j}^{L^{(k)}}}{\sqrt{\sum_{i=1}^{m}\left(a_{i j}^{U^{(k)}}\right)^{2}}}, \\
& b_{i j}^{U^{(k)}}=\frac{a_{i j}^{U^{(k)}}}{\sqrt{\sum_{i=1}^{m}\left(a_{i j}^{L^{(k)}}\right)^{2}}} .
\end{aligned}
$$

For the cost type, the standardizing formulae are listed as follows:

$$
\begin{aligned}
& b_{i j}^{L^{(k)}}=\frac{\left(1 / a_{i j}^{U^{(k)}}\right)}{\sqrt{\sum_{i=1}^{m}\left(1 / a_{i j}^{L^{(k)}}\right)^{2}}}, \\
& b_{i j}^{U^{(k)}}=\frac{\left(1 / a_{i j}^{L^{(k)}}\right)}{\sqrt{\sum_{i=1}^{m}\left(1 / a_{i j}^{U^{(k)}}\right)^{2}}} .
\end{aligned}
$$

Standardized interval number can be transformed into intuitionistic fuzzy numbers $\alpha_{i j}^{(k)}=\left(\mu_{i j}^{(k)}, v_{i j}^{(k)}, \pi_{i j}^{(k)}\right)$.

$$
\begin{gathered}
\mu_{i j}^{(k)}=b_{i j}^{L^{(k)}}, \quad v_{i j}^{(k)}=1-b_{i j}^{U^{(k)}}, \\
\pi_{i j}^{(k)}=b_{i j}^{U^{(k)}}-b_{i j}^{L^{(k)}} .
\end{gathered}
$$

TABLE 1: Linguistic variables for the importance of decision makers.

\begin{tabular}{lc}
\hline Linguistic variables & IFNs \\
\hline Very important & $(0.90,0.10,0.00)$ \\
Important & $(0.75,0.20,0.05)$ \\
Medium & $(0.50,0.45,0.05)$ \\
Unimportant & $(0.35,0.60,0.05)$ \\
Very unimportant & $(0.10,0.90,0.00)$ \\
\hline
\end{tabular}

TABLE 2: Conversion between linguistic variables and IFNs.

\begin{tabular}{lc}
\hline Linguistic variables & IFNs \\
\hline Extremely good (EG)/extremely high (EH) & $(1.00,0.00,0.00)$ \\
Very very good (VVG)/very very high (VVH) & $(0.90,0.10,0.00)$ \\
Very good (VG)/very high (VH) & $(0.80,0.10,0.10)$ \\
Good $(\mathrm{G}) /$ high $(\mathrm{H})$ & $(0.70,0.20,0.10)$ \\
Medium good (MG)/medium high (MH) & $(0.60,0.30,0.10)$ \\
Fair (F)/medium (M) & $(0.50,0.40,0.10)$ \\
Moderately poor $(\mathrm{MP}) /$ moderately low $(\mathrm{ML})$ & $(0.40,0.50,0.10)$ \\
Poor $(\mathrm{P}) /$ low $(\mathrm{L})$ & $(0.25,0.60,0.15)$ \\
Very poor $(\mathrm{VP}) /$ very low (VL) & $(0.10,0.75,0.15)$ \\
Very very poor $(\mathrm{VVP}) /$ very very low (VVL) & $(0.10,0.90,0.00)$ \\
\hline
\end{tabular}

(3) Conversion between linguistic variables and IFNs. A linguistic variable is variable whose values are words or sentences in natural or artificial language $[15,37]$. It is very useful in dealing with situations which are too complex or too ill-defined to be described properly in conventional quantitative expressions. For example, the subjective judgment of decision makers for the importance of the decision makers can all be expressed as linguistic variables such as very good, good, fair, poor, and very poor. Such linguistic variables can be converted into IFNs. The linguistic variables for the importance of the decision makers can be expressed in IFNs in Table 1. The ratings of alternatives with respect to the criteria can be converted into IFNs in Table 2. Table 2 shows the linguistic terms (given as in column 1) and their corresponding IFNs (given as in column 2) for decision makers to make assessments about the performance rating of each IS project alternative with respect to a given criterion.

2.3. Intuitionistic Fuzzy Entropy. As an important topic in the theory of fuzzy sets, entropy measures of IFSs have been investigated widely by many researchers from different points of view. de Luca and Termini [38] first introduced a nonprobabilistic entropy for fuzzy sets and formulated axioms with which the fuzzy entropy measure should comply. Burillo and Bustince [39] defined the distance measure between intuitionistic fuzzy sets and gave the axiom definition of intuitionistic fuzzy entropy and the theorem which characterized it. Szmidt and Kacprzyk [40] proposed a nonprobabilistictype entropy measure with a geometric interpretation of intuitionistic fuzzy sets. Hung and Yang [41] exploited the concept of probability to define the fuzzy entropy of intuitionistic fuzzy sets and proposed two families of entropy measures for IFSs and also constructed the axiom definition 
and properties. Many authors also proposed different entropy formulas for IFS $[21,42]$.

In this section we will introduce a formula given by Wang and Lei to calculate the entropy of an IFS.

Definition 5 (see [40]). A real-valued function $E: \operatorname{IFS}_{S}(X) \rightarrow$ $[0,1]$ is called an entropy for $\operatorname{IFS}_{S}(X)$, if it satisfies the following axiomatic requirements:

(1) $E(A)=0$ if and only if $A$ is a crisp set;

(2) $E(A)=1$ if and only if $\mu_{A}(x)=\nu_{A}(x)$ for each $\forall x \in$ $X$

(3) $E(A)=E\left(A^{c}\right)$ for each $\forall A \in \operatorname{IFS}_{S}(X)$;

(4) $E(A) \leq E(B)$ if $A$ is less fuzzy than $B$, that is, $\mu_{A}(x) \leq$ $\mu_{B}(x)$ and $v_{A}(x) \geq v_{B}(x)$ for $\mu_{B}(x) \leq v_{B}(x)$ or $\mu_{A}(x) \geq$ $\mu_{B}(x)$ and $\nu_{A}(x) \leq v_{B}(x)$ for $\mu_{B}(x) \geq v_{B}(x)$.

Definition 6 (see [42]). Let $X=\left(x_{1}, x_{2}, \ldots, x_{n}\right)$, and let $A=$ $\left\{\left\langle x, \mu_{A}(x), v_{A}(x)\right\rangle \mid x \in X\right\}$ be an IFS, and let the entropy of IFS $A$ be

$$
E(A)=\frac{1}{n} \sum_{i=1}^{n} \frac{\min \left(\mu_{A}(x), v_{A}(x)\right)+\pi_{A}(x)}{\max \left(\mu_{A}(x), v_{A}(x)\right)+\pi_{A}(x)} .
$$

Specially, for an intuitionistic fuzzy number $\alpha=$ $\left\langle\mu_{A}(x), v_{A}(x)\right\rangle$, the intuitionistic fuzzy entropy is given as follows:

$$
E(\alpha)=\frac{\min \left(\mu_{A}(x), v_{A}(x)\right)+\pi_{A}(x)}{\max \left(\mu_{A}(x), v_{A}(x)\right)+\pi_{A}(x)} .
$$

\section{A Hybrid MCGDM Approach for IS Project Selection Based on Intuitionistic Fuzzy Theory and TOPSIS Method}

3.1. The Procedure for IS Project Selection. Modeled as a MCGDM problem, the evaluation and selection of IS projects usually involve the following six steps as shown in Figure 1: (a) identifying the available IS projects as decision alternatives $A_{i}(i=1,2, \ldots, m)$ and the evaluation criteria $C_{j}(j=$ $1,2, \ldots, n)$, (b) constructing the hybrid decision matrix of each decision maker and transform hybrid multiple criteria matrix into intuitionistic fuzzy matrix, (c) determining the importance of decision makers, (d) constructing an aggregated intuitionistic fuzzy decision matrix based on the opinions of decision makers, (e) obtaining the entropy weights of the criteria, and (f) selecting the most preferred alternative as the most suitable IS project.

Information system (IS) project selection should be considered as a process that includes several related steps, the first important thing is to determine available IS projects and selection criteria, this step defines the multicriteria group decision making problem. Jiang and Klein [43] constructed a strategic grid model to explore the critical selection criteria. The six subcategories of IS evaluation criteria are included, which are financial, organizational, competing environment, technical, project risk, and managerial concerns. The importance of IS project relied heavily on internal organizational

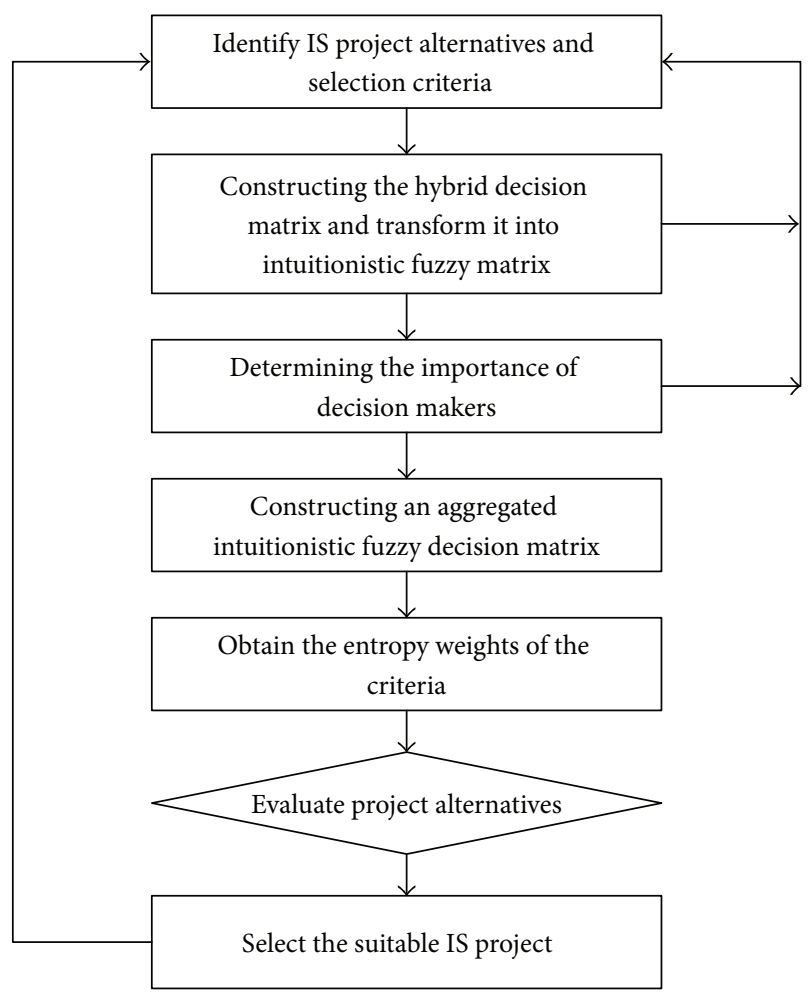

FIGURE 1: The procedure for IS project evaluation and selection.

goals, management support, and external environmental factors. Santhanam and Kyparisis [44, 45] identified several factors including corporate priorities, financial benefits and costs, and intangible measures such as project risk, availability of IS resources, and interdependencies among projects in the selection decision. Bradri et al. [9] formulated a mixed 0-1 goal programming model for IS projects selection decision which contained multiple factors including benefits, hardware, software, related costs, risk factors, preferences of decision makers and users for certain projects, cost of additional manpower required, completion time and training time constraints, and mutually exclusive and contingency requirements. In a practical selection, Chen and Gorla [46] employed fuzzy logic to incorporate qualitative criteria in an IS project selection model, which smoothly aids decision makers dealing with uncertain or incomplete information without losing existing quantitative information.

The next two steps involve constructing the hybrid decision matrix, then transforming it into intuitionistic fuzzy matrix and determin the importance of decision makers, which are to be addressed by the method presented in the next section. The IS project evaluation information given by the decision maker (or expert) over the alternatives under each criterion can be described by exact numbers, interval numbers, triangular fuzzy numbers, linguistic labels, or intuitionistic fuzzy numbers. The hybrid decision matrix can be transformed into intuitionistic fuzzy matrix which is a very suitable tool to deal with the imprecise or uncertain decision information. To model the uncertainty and subjectiveness of the human decision making process, linguistic terms are used 
for facilitating the subjective assessment to be made by the decision maker. These linguistic terms were assigned to IFNs by using the relationships presented in Table 1 . The fourth step is to construct an aggregated intuitionistic fuzzy decision matrix. In group decision making process, all the individual decision opinions need to be fused into a group opinion to construct an aggregated intuitionistic fuzzy decision matrix. Then based on the concept of intuitionistic fuzzy entropy, the weights of the $j$ th criteria can be calculated. The last step is to select the most preferred IS project based on the outcome of the method as follows.

3.2. A Hybrid MCGDM Approach for IS Project Selection Based on Intuitionistic Fuzzy Theory and TOPSIS Method. In this section, we discuss how to utilize the intuitionistic fuzzy entropy to identify the weight vector of the criteria in a hybrid MCGDM process for IS project selection. IS project selection problems are defined on a set of alternatives, from which the decision makers have to select the best alternative according to some criteria. Suppose that there exists an alternative set $A=\left\{A_{1}, A_{2}, \ldots, A_{n}\right\}$ which consists of $n$ alternatives, the decision makers will choose the best one from $A$ according to the criteria set $C=\left\{c_{1}, c_{2}, \ldots, c_{m}\right\}$ which includes $m$ criteria. For convenience, we denote the weight vector of criteria by $w=\left(w_{1}, w_{2}, \ldots, w_{m}\right)^{T}$, where $w_{j} \geq 0(j=1,2, \ldots, m)$ and $\sum_{j=1}^{m} w_{j}=1$.

Step 1 (construct the hybrid decision matrix $R^{(k)}(k=1,2,3)$ of each decision maker). In order to evaluate the candidate information system in a manageable and reliable way, decision makers rate the performance of each alternative with respect to the criteria previously identified. Each decision maker is asked to carefully rate the support provided by each project on each criterion. The hybrid decision matrix involves four different data types: exact values, intervals, IFVs, and linguistic variables; we can transform a hybrid decision matrix into intuitionistic fuzzy matrix according to the intuitionistic fuzzy transformation technique.

Assume that the rating of alternative $A_{i}$ with respect to critirion $c_{j}$ given by the $k$ th decision makers $d_{k}$ can be expressed in $\alpha_{i j}^{(k)}=\left(\mu_{i j}^{(k)}, v_{i j}^{(k)}, \pi_{i j}^{(k)}\right)$. Hence, the MCGDM problem can be concisely expressed in matrix format as follows:

$$
\widetilde{R}^{(k)}=\left(\alpha_{i j}^{(k)}\right)_{m \times n}=\left[\begin{array}{cccc}
\alpha_{11}^{(k)} & \alpha_{12}^{(k)} & \cdots & \alpha_{1 n}^{(k)} \\
\alpha_{21}^{(k)} & \alpha_{22}^{(k)} & \cdots & \alpha_{2 n}^{(k)} \\
\vdots & \vdots & & \vdots \\
\alpha_{m 1}^{(k)} & \alpha_{m 2}^{(k)} & \cdots & \alpha_{m n}^{(k)}
\end{array}\right]
$$

where $\alpha_{i j}^{(k)}=\left(\mu_{i j}^{(k)}, v_{i j}^{(k)}, \pi_{i j}^{(k)}\right)$.

Step 2. Calculate the weight with respect to the $k$ th decision maker $d_{k}$. The importance of the decision makers is consid- ered as linguistic variables, which can be expressed in IFNs in Table 2. Let $D_{k}=\left(\mu_{k}, v_{k}, \pi_{k}\right)$ be an intuitionistic fuzzy number for rating of the $k$ th decision maker. Then the weight $\lambda_{k}$ of the $k$ th decision maker can be obtained as follows [22]:

$$
\lambda_{k}=\frac{\left(\mu_{k}+\pi_{k}\left(\mu_{k} /\left(\mu_{k}+v_{k}\right)\right)\right)}{\sum_{k=1}^{t}\left(\mu_{k}+\pi_{k}\left(\mu_{k} /\left(\mu_{k}+\nu_{k}\right)\right)\right)},
$$

where $\sum_{k=1}^{t} \lambda_{k}=1$.

Step 3 (compose the aggregated intuitionistic fuzzy decision matrix). In this step, the aggregated intuitionistic fuzzy decision matrix (AIFDM) $R$, is composed by considering the intuitionistic fuzzy decision matrix given by decision makers and the vector of the decision maker weights. By considering weights $\lambda_{k}(k=1,2, \ldots, t)$ of decision makers, elements $\gamma_{i j}$ of the AIFDM can be calculated using IFWA as follows:

$$
\begin{aligned}
\gamma_{i j} & =\operatorname{IFWA}_{w_{t}}\left(\alpha_{i j}^{(1)}, \alpha_{i j}^{(2)}, \ldots, \alpha_{i j}^{(t)}\right) \\
& =\lambda_{1} \alpha_{i j}^{(1)} \oplus \lambda_{2} \alpha_{i j}^{(2)} \oplus \cdots \oplus \lambda_{t} \alpha_{i j}^{(t)} \\
& =\left(1-\prod_{k=1}^{t}\left(1-\mu_{i j}^{(k)}\right)^{\lambda_{t}}, \prod_{k=1}^{t}\left(1-v_{i j}^{(k)}\right)^{\lambda_{t}}\right),
\end{aligned}
$$

where $R=\left(\gamma_{i j}\right)_{n \times m}=\left(\left(\mu_{i j}^{\prime}, v_{i j}^{\prime}\right)\right)_{n \times m}, \lambda_{i}$ is the weight of $\alpha_{i}, \lambda_{i} \in$ $[0,1]$, and $\sum_{i=1}^{n} \lambda_{i}=1$.

Step 4 (determine the weights of the selection criteria). In this step, all criteria may not be assumed to be of equal importance. Let $w_{j}$ be the weights of the criteria. In order to obtain $w_{j}$, intuitionistic fuzzy entropy will be used firstly as follows:

$$
H_{j}=\frac{1}{n} \sum_{i=1}^{n} \frac{\min \left(\mu_{i j}^{\prime}, v_{i j}^{\prime}\right)+\pi_{i j}^{\prime}}{\max \left(\mu_{i j}^{\prime}, v_{i j}^{\prime}\right)+\pi_{i j}^{\prime}} .
$$

The entropy weights of the $j$ th criteria can be calculated as follows:

$$
w_{j}=\frac{1-H_{j}}{m-\sum_{j=1}^{m} H_{j}} .
$$

Step 5 (compute the intuitionistic fuzzy positive ideal solution and the intuitionistic fuzzy negative ideal solution). In order to apply TOPSIS, the intuitionistic fuzzy positive ideal solution (IFPIS) $\mathrm{M}^{+}$and the intuitionistic fuzzy negative ideal solution (IFNIS) $\mathrm{M}^{-}$have to be determined. Both solutions are vectors of IFN elements and they are derived from the AIFDM as follows. Let $B$ and $C$ be benefit and cost criteria, respectively. Then $M^{+}$and $M^{-}$are equal to the following:

$$
M^{+}=\left(\left(\mu_{i 1}^{\prime+}, v_{i 1}^{\prime+}\right),\left(\mu_{i 2}^{\prime+}, v_{i 2}^{\prime+}\right), \ldots,\left(\mu_{i m}^{\prime+}, v_{i m}^{\prime+}\right)\right) \text {, }
$$


where

$$
\begin{array}{r}
\mu_{i j}^{\prime+}=\left(\left(\max _{i} \mu_{i j}^{\prime}\left(x_{j}\right) \mid j \in B\right),\left(\min _{i} \mu_{i j}^{\prime}\left(x_{j}\right) \mid j \in C\right)\right), \\
v_{i j}^{\prime+}=\left(\left(\min _{i} v_{i j}^{\prime}\left(x_{j}\right) \mid j \in B\right),\left(\max _{i} v_{i j}^{\prime}\left(x_{j}\right) \mid j \in C\right)\right), \\
i=1,2, \ldots, n, \quad j=1, \ldots, m, \\
M^{-}=\left(\left(\mu_{i 1}^{\prime-}, v_{i 1}^{\prime-}\right),\left(\mu_{i 2}^{\prime-}, v_{i 2}^{\prime-}\right), \ldots,\left(\mu_{i m}^{\prime-}, v_{i m}^{\prime}\right)\right),
\end{array}
$$

where

$$
\begin{array}{r}
\mu_{i j}^{\prime-}=\left(\left(\min _{i} \mu_{i j}^{\prime}\left(x_{j}\right) \mid j \in B\right),\left(\max _{i} \mu_{i j}^{\prime}\left(x_{j}\right) \mid j \in C\right)\right), \\
v_{i j}^{\prime-}=\left(\left(\max _{i} v_{i j}^{\prime}\left(x_{j}\right) \mid j \in B\right),\left(\min _{i} v_{i j}^{\prime}\left(x_{j}\right) \mid j \in C\right)\right), \\
i=1,2, \ldots, n, \quad j=1, \ldots, m .
\end{array}
$$

Step 6 (calculate the weighted separation between the alternative IS project). Next, the separation measures $d^{+}$and $d^{-}$ are calculated for each candidate system $A_{i}$ from IFPIS and IFNIS, respectively. As a distance measure, the Hamming distance is adopted, since it has been proved to be a reliable distance measure that takes not only membership and nonmembership but also the hesitation part of IFNs into account. For each alternative these two weighted separation values can be calculated as follows:

$$
\begin{aligned}
& d^{+}=\frac{1}{2} \sum_{j=1}^{m} w_{j}\left(\left|\mu_{i j}^{\prime}-\mu_{j}^{+}\right|+\left|v_{i j}^{\prime}-v_{j}^{+}\right|+\left|\pi_{i j}^{\prime}-\pi_{j}^{+}\right|\right), \\
& d^{-}=\frac{1}{2} \sum_{j=1}^{m} w_{j}\left(\left|\mu_{i j}^{\prime}-\mu_{j}^{-}\right|+\left|v_{i j}^{\prime}-v_{j}^{-}\right|+\left|\pi_{i j}^{\prime}-\pi_{j}^{-}\right|\right) .
\end{aligned}
$$

Step 7 (calculate the relative closeness coefficient to the intuitionistic ideal solution). The final score of each system is derived by calculating the corresponding relative closeness coefficient with respect to the intuitionistic fuzzy ideal solution. For each alternative $A_{i}$, the relative closeness coefficient $k_{i}$ with respect to the IFPIS is defined as follows:

$$
k_{i}=\frac{d_{i}^{-}}{d_{i}^{+}+d_{i}^{-}} .
$$

Step 8 (rank all the alternatives). After the relatives closeness coefficient of each alternative is determined, the IS project proposals are ranked according to descending order of $k_{i}$. Once the projects are ranked according to their index value, the organization can select which project will be implemented. Naturally, the number of projects selected will depend on the designated budget.
TABLE 3: Hybrid decision matrix $R^{(1)}$ given by the decision maker $d_{1}$.

\begin{tabular}{ccccccc}
\hline & $c_{1}$ & $c_{2}$ & $c_{3}$ & $c_{4}$ & $c_{5}$ & $c_{6}$ \\
\hline$A_{1}$ & $\mathrm{MH}$ & {$[60,70]$} & 35 & $\mathrm{H}$ & $(0.45,0.35,0.20)$ & $\mathrm{VH}$ \\
$A_{2}$ & $\mathrm{VH}$ & {$[90,100]$} & 48 & $\mathrm{MH}$ & $(0.70,0.25,0.05)$ & $\mathrm{MH}$ \\
$A_{3}$ & $\mathrm{M}$ & {$[80,90]$} & 55 & $\mathrm{MH}$ & $(0.55,0.20,0.25)$ & $\mathrm{VH}$ \\
$A_{4}$ & $\mathrm{H}$ & {$[72,83]$} & 40 & $\mathrm{H}$ & $(0.60,0.15,0.25)$ & $\mathrm{M}$ \\
\hline
\end{tabular}

TABLE 4: Hybrid decision matrix $R^{(2)}$ given by the decision maker $d_{2}$.

\begin{tabular}{ccccccc}
\hline & $c_{1}$ & $c_{2}$ & $c_{3}$ & $c_{4}$ & $c_{5}$ & $c_{6}$ \\
\hline$A_{1}$ & $\mathrm{VH}$ & {$[62,75]$} & 36 & $\mathrm{H}$ & $(0.50,0.30,0.20)$ & $\mathrm{H}$ \\
$A_{2}$ & $\mathrm{H}$ & {$[85,95]$} & 47 & $\mathrm{MH}$ & $(0.60,0.25,0.15)$ & $\mathrm{MH}$ \\
$A_{3}$ & $\mathrm{MH}$ & {$[70,80]$} & 50 & $\mathrm{H}$ & $(0.55,0.15,0.30)$ & $\mathrm{VH}$ \\
$A_{4}$ & $\mathrm{MH}$ & {$[68,75]$} & 40 & $\mathrm{MH}$ & $(0.65,0.20,0.15)$ & $\mathrm{H}$ \\
\hline
\end{tabular}

TABle 5: Hybrid decision matrix $R^{(3)}$ given by the decision maker $d_{3}$.

\begin{tabular}{ccccccc}
\hline & $c_{1}$ & $c_{2}$ & $c_{3}$ & $c_{4}$ & $c_{5}$ & $c_{6}$ \\
\hline$A_{1}$ & $\mathrm{VH}$ & {$[70,80]$} & 40 & $\mathrm{MH}$ & $(0.45,0.15,0.40)$ & $\mathrm{H}$ \\
$A_{2}$ & $\mathrm{VH}$ & {$[90,95]$} & 60 & $\mathrm{H}$ & $(0.55,0.15,0.40)$ & $\mathrm{H}$ \\
$A_{3}$ & $\mathrm{MH}$ & {$[82,92]$} & 50 & $\mathrm{MH}$ & $(0.35,0.20,0.45)$ & $\mathrm{M}$ \\
$A_{4}$ & $\mathrm{H}$ & {$[68,78]$} & 43 & $\mathrm{H}$ & $(0.45,0.25,0.30)$ & $\mathrm{VH}$ \\
\hline
\end{tabular}

\section{Example Illustration and Discussion}

4.1. Example Illustration. In this section, we illustrate the approach by using a numerical example. Suppose that a high-technology manufacturing company desires to select an information system project to develop a new product. After initial screening, four candidates (i.e., alternatives) $A_{1}, A_{2}$, $A_{3}$, and $A_{4}$ remain for further evaluation. A committee of three decision makers $d_{1}, d_{2}$, and $d_{3}$ has been formed to conduct the interview and to select the most appropriate candidate.

Six criteria for IS project selection are considered, including outside environment conditions $\left(c_{1}\right)$, expected returns $\left(c_{2}\right)$, development costs $\left(c_{3}\right)$, project risks $\left(c_{4}\right)$, degree of ease to operate $\left(c_{5}\right)$, and management ability of the organization $\left(c_{6}\right)$. Criteria on development costs $\left(c_{3}\right)$ and project risks $\left(c_{4}\right)$ are cost criteria, and the others are benefit ones. The three decision makers $d_{k}(k=1,2,3)$ use the linguistic variables shown in Table 2 to evaluate the ratings of the alternatives $A_{i}$ $(i=1,2,3,4)$ with respect to the criteria $c_{j}(j=1,2,3, \ldots, 6)$ and construct, respectively, the decision matrix $R^{(k)}(k=$ $1,2,3$ ) as listed in Tables 3,4 , and 5 .

This is a hybrid MCGDM problem involving four different data types: exact values, intervals, linguistic terms, and IFNs. To solve this issue, we apply the developed method to the ranking and selection of the alternative IS project below. Three criteria including outside environment conditions $\left(c_{1}\right)$, project risks $\left(c_{4}\right)$ and management ability of the organization $\left(c_{6}\right)$ are expressed in the types of linguistic terms, criterion on expected returns $\left(c_{2}\right)$ is expressed in the interval forms, 
criterion on development costs $\left(c_{3}\right)$ is exact values and criterion on degree of ease to operate $\left(c_{5}\right)$ is expressed in the form of intuitionistic fuzzy numbers. In selection process, each decision maker expresses his/her preferences depending on the nature of the alternatives and on his/her own knowledge over them. The hybrid decision matries $R^{(1)}, R^{(2)}$, and $R^{(3)}$ are given by the decision maker $d_{1}, d_{2}$, and $d_{3}$, are shown as follows.

Step 1. Transform the hybrid decision matrix of each decision maker into intuitionistic fuzzy decision matrix. The exact values and intervals in the hybrid decision matrix given by the decision makers shown in Tables 3, 4, and 5 are standardized and then transformed into intuitionistic fuzzy numbers. The linguistic evaluation shown in Tables 3,4 , and 5 are converted into IFNs using Table 1 . Then, the intuitionistic fuzzy decision matrices $\widetilde{R}^{(k)}(k=1,2,3)$ of each decision maker shown in Tables 6,7 , and 8 are formed.

Step 2. Determine the weights of decision makers. The importance of the decision makers in the group decision making process is shown in Table 9. These linguistic variables used can be converted into IFNs by utilizing Table 2 . In order to obtain the weights $\lambda_{k}(k=1,2,3)$ of the decision makers, (16) is used as follows:

$$
\begin{aligned}
& \lambda_{1}=(0.90) \times\left(\left(0.75+0.05\left(\frac{0.75}{0.75+0.20}\right)\right)+0.90\right. \\
& \left.+\left(0.50+0.10\left(\frac{0.50}{0.50+0.40}\right)\right)\right)^{-1} \\
& =0.4009 \text {, } \\
& \lambda_{2}=\left(0.75+0.05\left(\frac{0.75}{0.75+0.20}\right)\right) \\
& \times\left(\left(0.75+0.05\left(\frac{0.75}{0.75+0.20}\right)\right)+0.90\right. \\
& \left.+\left(0.50+0.10\left(\frac{0.50}{0.50+0.40}\right)\right)\right)^{-1}=0.3516 \\
& \lambda_{3}=\left(0.50+0.10\left(\frac{0.50}{0.05+0.40}\right)\right) \\
& \times\left(\left(0.75+0.05\left(\frac{0.75}{0.75+0.20}\right)\right)+0.90\right. \\
& \left.+\left(0.50+0.10\left(\frac{0.50}{0.50+0.40}\right)\right)\right)^{-1}=0.2475 \text {. }
\end{aligned}
$$

Step 3. Construct the aggregated intuitionistic fuzzy decision matrix based on the opinions of decision makers. Utilize (17) of the IFWA operator to aggregate all the intuitionistic fuzzy decision matrix $\widetilde{R}^{(k)}(k=1,2,3)$ into a complex intuitionistic fuzzy decision matrix $R$ (Table 10).
Step 4. Obtain the entropy weights of the criteria. Utilize (18) to calculate the intuitionistic fuzzy entropy $H_{j}(j=$ $1,2, \ldots, 6)$ :

$$
\begin{array}{lll}
H_{1}=0.4170 ; & H_{2}=0.8420 ; & H_{3}=0.7610 ; \\
H_{4}=0.4612 ; & H_{5}=0.5771 ; & H_{6}=0.3702 .
\end{array}
$$

Then, using (19) to obtain the entropy weights:

$$
\begin{array}{lll}
w_{1}=0.2267 ; & w_{2}=0.0614 ; & w_{3}=0.0929 ; \\
w_{4}=0.2095 ; & w_{5}=0.1645 ; & w_{6}=0.2449 .
\end{array}
$$

Step 5. Obtain intuitionistic fuzzy positive-ideal solution and intuitionistic fuzzy negative-ideal solution by using (20)(22). Consider the following:

$$
\begin{aligned}
M^{+}= & (0.77,0.13,0.10),(0.52,0.35,0.13), \\
& (0.59,0.41,0.00),(0.68,0.27,0.10), \\
& (0.63,0.18,0.19),(0.72,0.17,0.11)), \\
M^{-}= & (0.56,0.34,0.10),(0.37,0.50,0.13), \\
& (0.42,0.58,0.00),(0.63,0.22,0.10), \\
& (0.47,0.27,0.26),(0.67,0.22,0.11)) .
\end{aligned}
$$

Step 6 (calculate the separation measures). Negative and positive separation measures based on normalized hamming distance for each alternative are calculated in Table 11 by utilizing (23)-(24).

Step 7 (rank the alternatives). The relative closeness coefficients are determined, and then four alternatives are ranked according to descending order of $k_{i}$. The alternatives are ranked as $A_{2}>A_{1}>A_{4}>A_{3}$. Information system project $A_{2}$ is selected as appropriate alternative.

4.2. Comparison and Discussion. IS project selection from many competing proposals is a critical business activity for all organizations. There are various methods proposed for solving IS project selection problems, such as the scoring approach, the ranking method, the AHP or ANP method, and the approach based on intuitionistic fuzzy theory.

To illustrate the effectiveness of the proposed hybrid MCGDM methods based on intuitionistic fuzzy theory, we used the above case study to analyze comparable methods, which include a combined intuitionistic fuzzy set-TOPSIS method (Method 1) [25] and an intuitionistic fuzzy optimum selecting model (Method 2) [30]. For the combined intuitionistic fuzzy set-TOPSIS method (Method 1), the weights of the selection criteria are determined by the users of IS project. Three users of IS project are asked to denote a grade for the importance of each criterion in a linguistic term, and the level of user expertise is also expressed in the form of linguistic variables, as it is shown in Table 12. The three users $U_{1}, U_{2}$, and $U_{3}$ also agree that their level of expertise in managing large projects can be characterized as "Master," "Expert," and "Proficient," respectively. Opinions of users $U_{1}, U_{2}$, and $U_{3}$ 
TABLE 6: Intuitionistic decision matrix $\widetilde{R}^{(1)}$.

\begin{tabular}{ccccccc}
\hline & $c_{1}$ & $c_{2}$ & $c_{3}$ & $c_{4}$ & $c_{5}$ & $c_{6}$ \\
\hline$A_{1}$ & $(0.60,0.30,0.10)$ & $(0.35,0.54,0.11)$ & $(0.61,0.39,0.00)$ & $(0.70,0.20,0.10)$ & $(0.45,0.35,0.20)$ & $(0.80,0.10,0.10)$ \\
$A_{2}$ & $(0.80,0.10,0.10)$ & $(0.52,0.34,0.14)$ & $(0.44,0.56,0.00)$ & $(0.60,0.30,0.10)$ & $(0.75,0.25,0.05)$ & $(0.60,0.30,0.10)$ \\
$A_{3}$ & $(0.50,0.40,0.10)$ & $(0.46,0.41,0.13)$ & $(0.39,0.61,0.00)$ & $(0.60,0.30,0.10)$ & $(0.55,0.20,0.25)$ & $(0.80,0.10,0.10)$ \\
$A_{4}$ & $(0.70,0.20,0.10)$ & $(0.42,0.46,0.12)$ & $(0.53,0.47,0.00)$ & $(0.70,0.20,0.10)$ & $(0.60,0.15,0.25)$ & $(0.50,0.40,0.10)$ \\
\hline
\end{tabular}

TABLE 7: Intuitionistic decision matrix $\widetilde{R}^{(2)}$.

\begin{tabular}{ccccccc}
\hline & $c_{1}$ & $c_{2}$ & $c_{3}$ & $c_{4}$ & $c_{5}$ & $c_{6}$ \\
\hline$A_{1}$ & $(0.80,0.10,0.10)$ & $(0.38,0.48,0.14)$ & $(0.59,0.41,0.00)$ & $(0.70,0.20,0.10)$ & $(0.50,0.30,0.20)$ & $(0.60,0.30,0.10)$ \\
$A_{2}$ & $(0.70,0.20,0.10)$ & $(0.52,0.34,0.14)$ & $(0.45,0.55,0.00)$ & $(0.60,0.30,0.10)$ & $(0.60,0.25,0.15)$ & $(0.80,0.10,0.10)$ \\
$A_{3}$ & $(0.60,0.30,0.10)$ & $(0.43,0.44,0.13)$ & $(0.42,0.58,0.00)$ & $(0.70,0.20,0.10)$ & $(0.55,0.15,0.30)$ & $(0.70,0.20,0.10)$ \\
$A_{4}$ & $(0.60,0.30,0.10)$ & $(0.42,0.48,0.10)$ & $(0.53,0.47,0.00)$ & $(0.60,0.30,0.10)$ & $(0.65,0.20,0.15)$ & $(0.70,0.20,0.10)$ \\
\hline
\end{tabular}

TABLE 8: Intuitionistic decision matrix $\widetilde{R}^{(3)}$.

\begin{tabular}{ccccccc}
\hline & $c_{1}$ & $c_{2}$ & $c_{3}$ & $c_{4}$ & $c_{5}$ & $c_{6}$ \\
\hline$A_{1}$ & $(0.80,0.10,0.10)$ & $(0.40,0.49,0.11)$ & $(0.58,0.42,0.00)$ & $(0.60,0.30,0.10)$ & $(0.45,0.15,0.40)$ & $(0.70,0.20,0.10)$ \\
$A_{2}$ & $(0.80,0.10,0.10)$ & $(0.52,0.39,0.09)$ & $(0.39,0.61,0.00)$ & $(0.70,0.20,0.10)$ & $(0.55,0.15,0.30)$ & $(0.70,0.20,0.10)$ \\
$A_{3}$ & $(0.60,0.30,0.10)$ & $(0.47,0.41,0.12)$ & $(0.46,0.53,0.00)$ & $(0.60,0.30,0.10)$ & $(0.35,0.20,0.45)$ & $(0.50,0.40,0.10)$ \\
$A_{4}$ & $(0.70,0.20,0.10)$ & $(0.39,0.50,0.11)$ & $(0.54,0.46,0.00)$ & $(0.70,0.20,0.10)$ & $(0.45,0.25,0.30)$ & $(0.80,0.10,0.10)$ \\
\hline
\end{tabular}

TABLE 9: The importance of decision makers.

\begin{tabular}{lc}
\hline & Linguistic variables \\
\hline$d_{1}$ & Very important \\
$d_{2}$ & Important \\
$d_{3}$ & Medium \\
\hline
\end{tabular}

on the importance of the criteria are presented in columns of Table 13 entitled with the label "Users". These opinions are assigned to corresponding IFNs by using the relationships shown in Table 12. For intuitionistic fuzzy optimum selecting model (Method 2), the concept of intuitionistic fuzzy entropy defined by Wang and Lei is used to determine the weights of the selection criteria.

Table 14 exhibits the ranking comparisons of all the four alternatives as obtained using these approaches. We can see that the ranking results of the proposed approach and the comparable methods are the same. This proved that the proposed method is particularly powerful and pragmatic. It is an excellent way to solve IS project selections.

Although the approach presented here is so effective, however, it is still simple and do not contain sufficient information for evaluation. For a proper IS project selection, it is necessary to enrich not only the evaluation criteria but also the selection methodology. Here only six criteria are considered, but these criteria alone are not sufficient to provide adequate information. Furthermore, priority of the criteria for IS project selection may be different in different organization. Therefore, detailed studies are needed to find other criteria or attributes of effective IS project.

Concerning the selection methodology, this paper uses the intuitionistic fuzzy weighted average (IFWA) operator to fuse the decision information. Information fusion has played an important role in the field of MCGDM. Because the IFSs are highly useful in handling fuzziness and uncertainty in practical applications, recently, a variety of aggregation operators have been developed and used to fuse the decision information in MCGDM so as to find the optimal decision alternative. These aggregation operators include the intuitionistic fuzzy weighted averaging (IFWA) operator, the intuitionistic fuzzy ordered weighted averaging (IFOWA) operator, the dynamic intuitionistic fuzzy weighted averaging (DIFWA) operator, the intuitionistic fuzzy weighted geometric (IFWG) operator, the intuitionistic fuzzy ordered weighted geometric operator, the dynamic intuitionistic fuzzy weighted geometric (DIFWG) operator, the intuitionistic fuzzy hybrid aggregation (IFHA) operator, and some other operators. To select the appropriate project and promote continuing research in future, it would be worthwhile to use these aggregation operators like the intuitionistic fuzzy ordered weighted averaging (IFOWA) operator, the dynamic intuitionistic fuzzy weighted averaging (DIFWA) operator, and so forth.

\section{Conclusion}

Evaluating and selecting IS projects are complex processes, as they often involve multiple decision makers making subjective and imprecise assessments in relation to multiple IS project alternatives and multiple evaluation criteria. This paper presents a hybrid MCGDM approach for evaluation of IS project using intuitionistic fuzzy theory and TOPSIS method. Intuitionistic fuzzy sets are suitable way to deal with uncertainty. In the evaluation process, the ratings of each alternative with respect to each criterion are given in 
TABLE 10: Intuitionistic decision matrix $R$.

\begin{tabular}{ccccccc}
\hline & $c_{1}$ & $c_{2}$ & $c_{3}$ & $c_{4}$ & $c_{5}$ \\
\hline$A_{1}$ & $(0.74,0.16,0.10)$ & $(0.37,0.50,0.13)$ & $(0.59,0.41,0.00)$ & $(0.68,0.22,0.10)$ & $(0.47,0.27,0.26)$ & $(0.72,0.17,0.11)$ \\
$A_{2}$ & $(0.77,0.13,0.10)$ & $(0.52,0.35,0.13)$ & $(0.43,0.57,0.00)$ & $(0.63,0.27,0.10)$ & $(0.63,0.22,0.15)$ & $(0.71,0.18,0.11)$ \\
$A_{3}$ & $(0.56,0.34,0.10)$ & $(0.45,0.42,0.13)$ & $(0.42,0.58,0.00)$ & $(0.64,0.26,0.10)$ & $(0.51,0.18,0.31)$ & $(0.71,0.18,0.11)$ \\
$A_{4}$ & $(0.67,0.23,0.10)$ & $(0.41,0.47,0.12)$ & $(0.53,0.47,0.00)$ & $(0.67,0.23,0.10)$ & $(0.59,0.19,0.22)$ & $(0.67,0.22,0.11)$ \\
\hline
\end{tabular}

TABLE 11: Separation measures and the relative closeness coefficient of each alternative.

\begin{tabular}{lcccc}
\hline Alternatives & $d_{i}^{+}$ & $d_{i}^{-}$ & $k_{i}$ & Ranking \\
\hline$A_{1}$ & 0.0493 & 0.0751 & 0.6036 & 2 \\
$A_{2}$ & 0.0293 & 0.1003 & 0.7742 & 1 \\
$A_{3}$ & 0.0956 & 0.0356 & 0.2712 & 4 \\
$A_{4}$ & 0.0618 & 0.0617 & 0.4996 & 3 \\
\hline
\end{tabular}

TABLE 12: Linguistic variables for the importance of users and criteria.

\begin{tabular}{lcr}
\hline $\begin{array}{l}\text { Level of user } \\
\text { expertise }\end{array}$ & $\begin{array}{c}\text { Importance of selection } \\
\text { criteria }\end{array}$ & \multicolumn{1}{c}{ IFNs } \\
\hline Master & Very important (VI) & $(0.90,0.10,0.00)$ \\
Expert & Important (I) & $(0.75,0.20,0.05)$ \\
Proficient & Medium (M) & $(0.45,0.05)$ \\
Practitioner & Unimportant (UI) & $(0.35,0.60,0.05)$ \\
Beginner & Very unimportant (VU) & $(0.10,0.90,0.00)$ \\
\hline
\end{tabular}

TABLE 13: Importance of values and weights of the criteria.

\begin{tabular}{lcccccc}
\hline Criteria & Users & & & \multicolumn{2}{c}{ Weights } \\
& $U_{1}$ & $U_{2}$ & $U_{3}$ & $\mu$ & 0.1000 \\
\hline$c_{1}$ & $\mathrm{VI}$ & $\mathrm{VI}$ & $\mathrm{VI}$ & 0.9000 & 0.3384 & 0.0000 \\
$c_{2}$ & $\mathrm{M}$ & $\mathrm{I}$ & $\mathrm{M}$ & 0.6081 & 0.3251 & 0.0535 \\
$c_{3}$ & $\mathrm{I}$ & $\mathrm{M}$ & $\mathrm{M}$ & 0.6213 & 0.2768 & 0.0536 \\
$c_{4}$ & $\mathrm{M}$ & $\mathrm{I}$ & $\mathrm{I}$ & 0.6699 & 0.2660 & 0.0532 \\
$c_{5}$ & $\mathrm{I}$ & $\mathrm{M}$ & $\mathrm{I}$ & 0.6810 & 0.1515 & 0.0217 \\
$c_{6}$ & $\mathrm{VI}$ & $\mathrm{I}$ & $\mathrm{I}$ & 0.8269 & & 0.0530 \\
\hline
\end{tabular}

TABLE 14: Ranking comparisons.

\begin{tabular}{lccc}
\hline Alternatives & Proposed approach & Method 1 & Method 2 \\
\hline$A_{1}$ & 2 & 2 & 2 \\
$A_{2}$ & 1 & 1 & 1 \\
$A_{3}$ & 4 & 4 & 4 \\
$A_{4}$ & 3 & 3 & 3 \\
\hline
\end{tabular}

the different forms such as exact values, intervals, IFVs, or linguistic terms and the importance of the decision makers is expressed as linguistic variables. All these evaluation pieces of information can be transformed to the form of intuitionistic fuzzy numbers. Also intuitionistic fuzzy averaging operator is utilized to aggregate opinions of decision makers. Intuitionistic fuzzy entropy is utilized to aggregate individual opinions of decision makers and can be used to obtain the entropy weights of the criteria, respectively.
After intuitionistic fuzzy positive-ideal solution and intuitionistic fuzzy negative-ideal solution are calculated based on the weighted hamming distance, the relative closeness coefficients of alternatives are obtained and alternatives are ranked. The approach developed above can be more useful for solving practical IS project selection problems. With its simplicity in concept and computation, the method is applicable to the general multicriteria evaluation and selection problem. We suggest that future research studies could incorporate more criteria in order to make more accurate analysis. Furthermore, some other aggregation operators could be used to fuse intuitionistic fuzzy information in the future.

\section{Conflict of Interests}

The author declares that there is no conflict of interests regarding the publication of this paper. 


\section{Acknowledgment}

This work was supported by the National Natural Science Foundation of China under Grant no. 70901079.

\section{References}

[1] H. Deng and S. Wibowo, "Intelligent decision support for evaluating and selecting information systems projects," Engineering Letters, vol. 16, no. 3, pp. 412-418, 2008.

[2] C.-H. Yeh, H. Deng, S. Wibowo, and Y. Xu, "Fuzzy multicriteria decision support for information systems project selection," International Journal of Fuzzy Systems, vol. 12, no. 2, pp. 170179, 2010.

[3] C.-T. Chen and H.-L. Cheng, "A comprehensive model for selecting information system project under fuzzy environment," International Journal of Project Management, vol. 27, no. 4, pp. 389-399, 2009.

[4] J. L. King and E. L. Schrems, "Cost-benefit analysis in information systems development and operation," ACM Computing Surveys, vol. 10, no. 1, pp. 19-34, 1978.

[5] H. C. Lucas and J. R. Moore, "A multiple-criterion scoring approach to information system project selection," Information, vol. 14, no. 1, pp. 1-12, 1976.

[6] M. D. Buss, "How to rank computer projects," Harvard Business Review, vol. 61, no. 1, pp. 118-125, 1983.

[7] J. D. McKeen and T. Guimaraes, "Selecting mis projects by steering committee," Communications of the ACM, vol. 28, no. 12, pp. 1344-1352, 1985.

[8] K. Muralidhar, R. Santhanam, and R. L. Wilson, "Using the analytic hierarchy process for information system project selection," Information and Management, vol. 18, no. 2, pp. 87-95, 1990.

[9] M. A. Badri, D. Davis, and D. Davis, "A comprehensive 0-1 goal programming model for project selection," International Journal of Project Management, vol. 19, pp. 243-252, 2001.

[10] S. Nalchigar and S. M. Nasserazdeh, "Application of DEA for selecting most efficient information system project with imprecise data," International Journal of Mathematical Modeling and Computations, vol. 1, no. 1, pp. 15-25, 2011.

[11] J. W. Lee and S. H. Kim, "Integrated approach for interdependent information system project selection," International Journal of Project Management, vol. 19, no. 2, pp. 111-118, 2001.

[12] W. W. Wu, "A hybrid approach to IT project selection," WSEAS Transactions on Business and Economics, vol. 5, no. 6, pp. 361371, 2008.

[13] C. E. Bozdag, C. Kahraman, and D. Ruan, "Fuzzy group decision making for selection among computer integrated manufacturing systems," Computers in Industry, vol. 51, no. 1, pp. 13-29, 2003.

[14] L. A. Zadeh, "Fuzzy sets," Information and Computation, vol. 8, pp. 338-353, 1965.

[15] M. Delgado, J. L. Verdegay, and M. A. Vila, "Linguistic decisionmaking models," International Journal of Intelligent Systems, vol. 7, no. 5, pp. 479-492, 1992.

[16] K. T. Atanassov, "Intuitionistic fuzzy sets," Fuzzy Sets and Systems, vol. 20, no. 1, pp. 87-96, 1986.

[17] S. K. De, R. Biswas, and A. R. Roy, "An application of intuitionistic fuzzy sets in medical diagnosis," Fuzzy Sets and Systems, vol. 117, no. 2, pp. 209-213, 2001.
[18] D.-F. Li, "Multiattribute decision making models and methods using intuitionistic fuzzy sets," Journal of Computer and System Sciences, vol. 70, no. 1, pp. 73-85, 2005.

[19] D.-F. Li, "Extension of the LINMAP for multiattribute decision making under Atanassov's intuitionistic fuzzy environment," Fuzzy Optimization and Decision Making, vol. 7, no. 1, pp. 1734, 2008.

[20] L. Dengfeng and C. Chuntian, "New similarity measures of intuitionistic fuzzy sets and application to pattern recognitions," Pattern Recognition Letters, vol. 23, no. 1-3, pp. 221-225, 2002.

[21] I. K. Vlachos and G. D. Sergiadis, "Intuitionistic fuzzy information-applications to pattern recognition," Pattern Recognition Letters, vol. 28, no. 2, pp. 197-206, 2007.

[22] F. E. Boran, S. Genç, M. Kurt, and D. Akay, "A multi-criteria intuitionistic fuzzy group decision making for supplier selection with TOPSIS method," Expert Systems with Applications, vol. 36, no. 8, pp. 11363-11368, 2009.

[23] F. Ye, "An extended TOPSIS method with interval-valued intuitionistic fuzzy numbers for virtual enterprise partner selection," Expert Systems with Applications, vol. 37, no. 10, pp. 7050-7055, 2010.

[24] S.-F. Zhang and S.-Y. Liu, "A GRA-based intuitionistic fuzzy multi-criteria group decision making method for personnel selection," Expert Systems with Applications, vol. 38, no. 9, pp. 11401-11405, 2011.

[25] V. C. Gerogiannis, P. Fitsilis, and A. D. Kameas, "Using a combined intuitionistic fuzzy set-TOPSIS method for evaluating project and portfolio management information systems," IFIP Advances in Information and Communication Technology, vol. 364, no. 2, pp. 67-81, 2011.

[26] C. Y. Liang, E. Q. Zhang, X. W. Qing, and Q. Lu, "A method of multi-attribute group decision making with incomplete hybrid assessment information," Chinese Journal of Management Science, vol. 17, no. 4, pp. 126-132, 2009.

[27] W. Wang and M. Cui, "Hybrid multiple attribute decision making model based on entropy," Journal of Systems Engineering and Electronics, vol. 18, no. 1, pp. 72-75, 2007.

[28] F. Herrera, L. Martínez, and P. J. Sánchez, "Managing nonhomogeneous information in group decision making," European Journal of Operational Research, vol. 166, no. 1, pp. 115-132, 2005.

[29] L. Martínez, J. Liu, D. Ruan, and J.-B. Yang, "Dealing with heterogeneous information in engineering evaluation processes," Information Sciences, vol. 177, no. 7, pp. 1533-1542, 2007.

[30] Y.-J. Si and F.-J. Wei, "Hybrid multi-attribute decision making based on the intuitionistic fuzzy optimum selecting model," Systems Engineering and Electronics, vol. 31, no. 12, pp. 28932897, 2009.

[31] C. Y. Liang, X. W. Qi, Y. Ding, and Y. J. Leng, "A hybrid multicriteria group decision making with TOPSIS method," Chinese Journal of Management Science, vol. 12, no. 4, pp. 126-132, 2012.

[32] K. H. Guo and W. L. Li, "An attitudinal-based method for constructing intuitionistic fuzzy information in hybrid MADM under uncertainty," Information Sciences, vol. 208, no. 15, pp. 28 38, 2012.

[33] X.-W. Chen, W.-S. Wang, G.-B. Song, and D.-Y. Song, "Hybrid multiattribute decision making based on fuzzy preference relation," Systems Engineering and Electronics, vol. 34, no. 3, pp. 529-533, 2012.

[34] Z. S. Xu and R. R. Yager, "Some geometric aggregation operators based on intuitionistic fuzzy sets," International Journal of General Systems, vol. 35, no. 4, pp. 417-433, 2006. 
[35] Z. S. Xu, "Intuitionistic fuzzy aggregation operators," IEEE Transactions on Fuzzy Systems, vol. 15, no. 6, pp. 1179-1187, 2007.

[36] E. Szmidt and J. Kacprzyk, "Distances between intuitionistic fuzzy sets," Fuzzy Sets and Systems, vol. 114, no. 3, pp. 505-518, 2000.

[37] V.-N. Huynh and Y. Nakamori, "A satisfactory-oriented approach to multiexpert decision-making with linguistic assessments," IEEE Transactions on Systems, Man, and Cybernetics B, vol. 35, no. 2, pp. 184-196, 2005.

[38] A. de Luca and S. Termini, "A definition of a nonprobabilistic entropy in the setting of fuzzy sets theory," Information and Computation, vol. 20, pp. 301-312, 1972.

[39] P. Burillo and H. Bustince, "Entropy on intuitionistic fuzzy sets and on interval-valued fuzzy sets," Fuzzy Sets and Systems, vol. 78, no. 3, pp. 305-316, 1996.

[40] E. Szmidt and J. Kacprzyk, "Entropy for intuitionistic fuzzy sets," Fuzzy Sets and Systems, vol. 118, no. 3, pp. 467-477, 2001.

[41] W.-L. Hung and M.-S. Yang, "Fuzzy entropy on intuitionistic fuzzy sets," International Journal of Intelligent Systems, vol. 21, no. 4, pp. 443-451, 2006.

[42] Y. Wang and Y.-J. Lei, "A technique for constructing intuitionistic fuzzy entropy," Control and Decision, vol. 22, no. 12, pp. 13901394, 2007.

[43] J. J. Jiang and G. Klein, "Project selection criteria by strategic orientation," Information and Management, vol. 36, no. 2, pp. 63-75, 1999.

[44] R. Santhanam and J. Kyparisis, "A multiple criteria decision model for information system project selection," Computers and Operations Research, vol. 22, no. 8, pp. 807-818, 1995.

[45] R. Santhanam and G. J. Kyparisis, "A decision model for interdependent information system project selection," European Journal of Operational Research, vol. 89, no. 2, pp. 380-399, 1996.

[46] K. Chen and N. Gorla, "Information system project selection using fuzzy logic," IEEE Transactions on Systems, Man, and Cybernetics Part A, vol. 28, no. 6, pp. 849-855, 1998. 


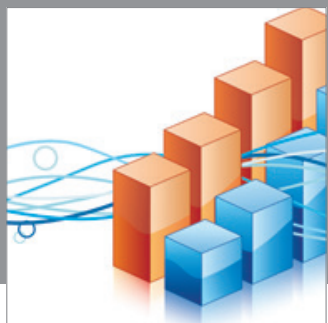

Advances in

Operations Research

mansans

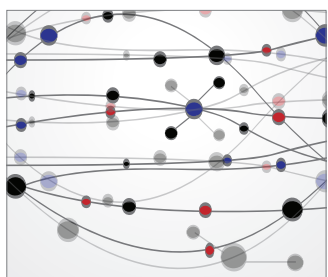

The Scientific World Journal
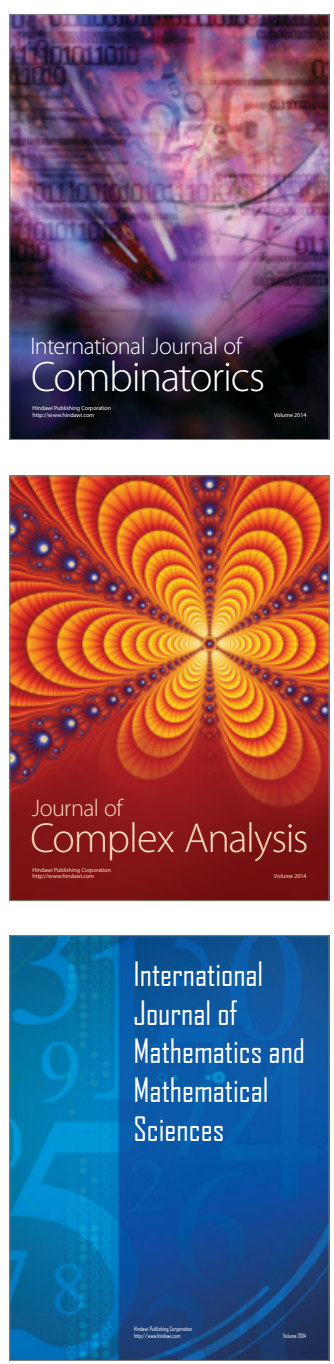
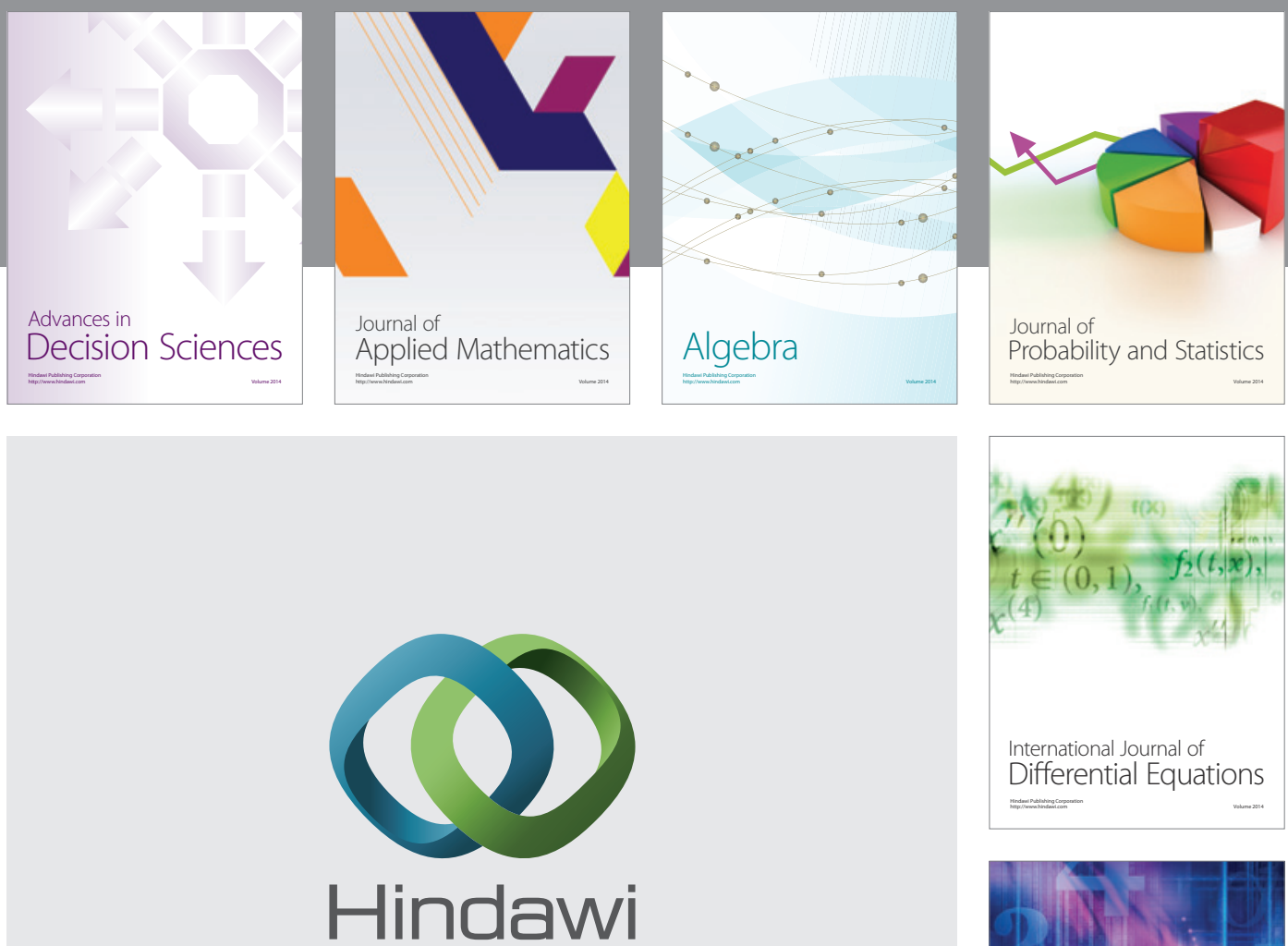

Submit your manuscripts at http://www.hindawi.com
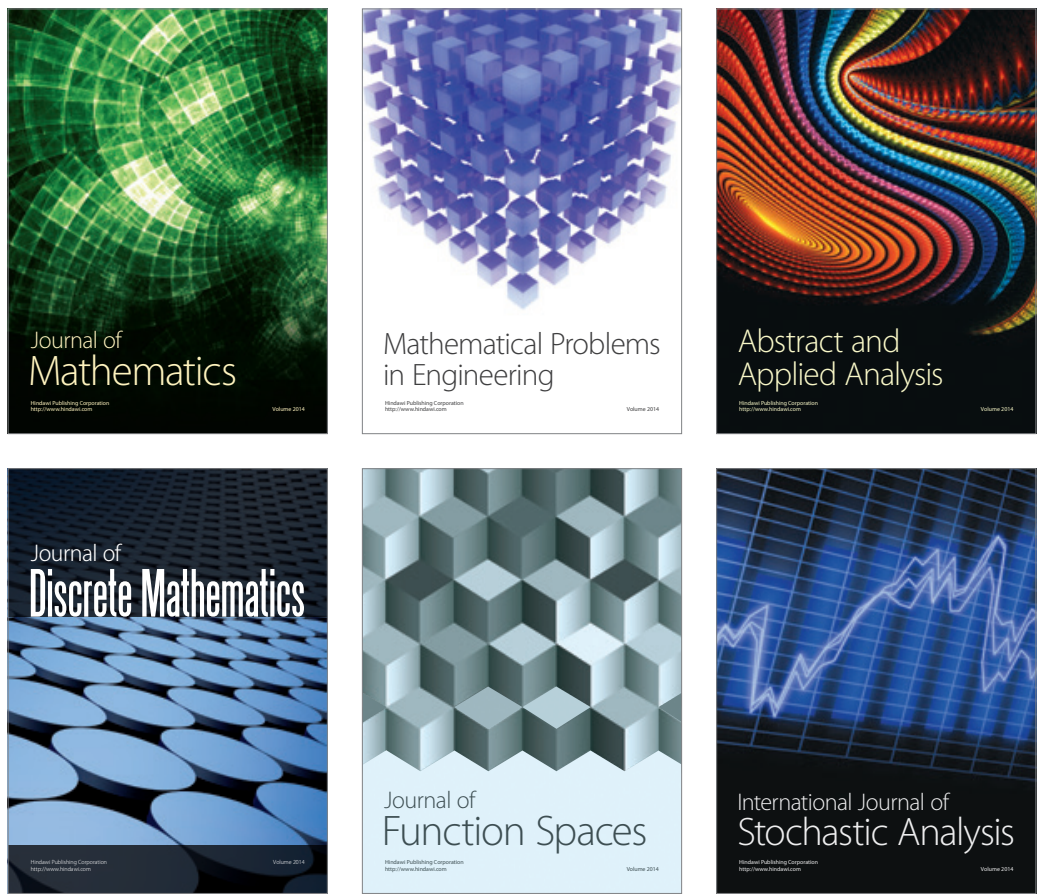

Journal of

Function Spaces

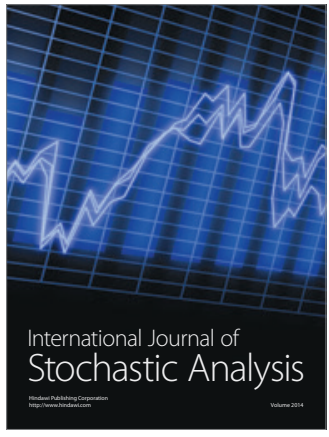

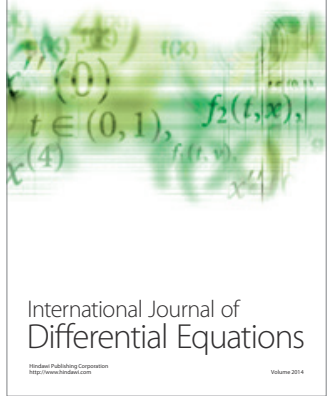
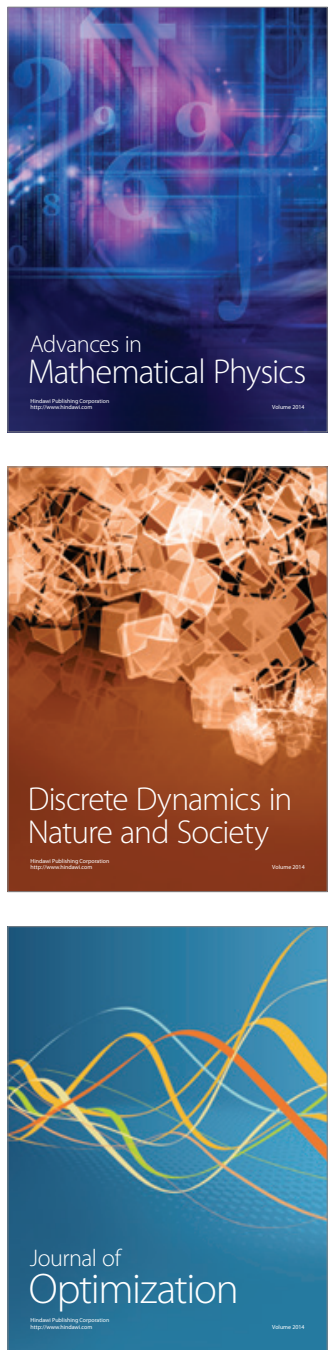